\title{
CHARACTERIZATIONS OF REGULAR LOCAL RINGS IN POSITIVE CHARACTERISTICS
}

\author{
JINJIA LI \\ (Communicated by Bernd Ulrich)
}

\begin{abstract}
In this note, we provide several characterizations of regular local rings in positive characteristics, in terms of the Hilbert-Kunz multiplicity and its higher Tor counterparts $i t_{i}=\lim _{n \rightarrow \infty} \ell\left(\operatorname{Tor}_{i}\left(k,{ }^{n} R\right)\right) / p^{n d}$. We also apply the characterizations to improve a recent result by Bridgeland and Iyengar in the characteristic $p$ case. Our proof avoids using the existence of big CohenMacaulay modules, which is the major tool in the proof of Bridgeland and Iyengar.
\end{abstract}

\section{INTRODUCTION}

Let $(R, \mathfrak{m}, k)$ be a $d$-dimensional local ring of characteristic $p>0$. The Frobenius endomorphism $f_{R}: R \rightarrow R$ is defined by $f_{R}(r)=r^{p}$ for $r \in R$. Each iteration $f_{R}^{n}$ defines a new $R$-module structure on $R$, denoted $f^{n} R$, for which $a \cdot b=a^{p^{n}} b$. For any $R$-module $M, F_{R}^{n}(M)$ stands for $M \otimes_{R} f^{n} R$, the $R$-module structure of which is given by the base change along the Frobenius endomorphism. When $M$ is a cyclic module $R / I$, it is easy to show that $F^{n}(R / I) \cong R / I^{\left[p^{n}\right]}$, where $I^{\left[p^{n}\right]}$ denotes the ideal generated by the $p^{n}$-th power of the generators of $I$.

In what follows, $\ell(-)$ denotes the length function.

For any m-primary ideal $I$, the Hilbert-Kunz multiplicity of $R$ with respect to $I$ was first introduced by Monsky in [Mo]:

$$
e_{\mathrm{HK}}(I, R)=\lim _{n \rightarrow \infty} \ell\left(F^{n}(R / I)\right) / p^{n d} .
$$

The Hilbert-Kunz multiplicity of $R$ is $e_{\mathrm{HK}}(R)=e_{\mathrm{HK}}(\mathfrak{m}, R)$. We also frequently write $e_{\mathrm{HK}}(I)=e_{\mathrm{HK}}(I, R)$. It has been shown by many authors that the HilbertKunz multiplicity encodes subtle information about the singularity of $R$. One such example is the following characterization of the regularity due to Watanabe and Yoshida; see [W-Y], [H-Y].

Theorem 1.1. If $R$ is unmixed, then it is regular if and only if $e_{H K}(R)=1$.

It is natural to ask whether the higher Tor counterparts of the Hilbert-Kunz multiplicity, which are defined below, can encode similar information on the singularity of the ring.

Received by the editors December 1, 2006 and, in revised form, February 19, 2007.

2000 Mathematics Subject Classification. Primary 13A35, 13D07, 13D25, 13 H05.

Key words and phrases. Regular local ring, Hilbert-Kunz multiplicity, Frobenius, Tor. 
Definition. Let $R$ be a $d$-dimensional local ring of characteristic $p>0$. Let $I$ be any $\mathfrak{m}$-primary ideal. Define

$$
\mathfrak{t}_{i}(I, R)=\lim _{n \rightarrow \infty} \ell\left(\operatorname{Tor}_{i}\left(R / I,{ }^{f^{n}} R\right)\right) / p^{n d} .
$$

Seibert has shown that such limits always exist [Se]. In the sequel, we also write $\mathfrak{t}_{i}(R)=\mathfrak{t}_{i}(\mathfrak{m}, R)$. The main result of this note is the following theorem in Section 2 :

Main Theorem (Theorem 2.2). Let $(R, \mathfrak{m}, k)$ be a d-dimensional local ring of characteristic $p>0$. Then the following are equivalent:

(i) $R$ is regular,

(ii) $\mathfrak{t}_{1}(R)=0$,

(iii) $\mathfrak{t}_{2}(R)=0$,

(iv) $e_{H K}(R)-1=\mathfrak{t}_{1}(R)$.

The proof of this theorem is inspired by the work of Huneke and Yao [H-Y, 2.1] and Blickle and Enescu [B-E].

In Section 3, we apply our main theorem to slightly generalize the positive characteristic case of a recent result by Bridgeland and Iyengar [B-I, 1.1]. The result of Bridgeland and Iyengar states that if $R$ contains a field and if $k$ is a direct summand of $H_{0}\left(C_{\bullet}\right)$, where $C_{\bullet}$ is a perfect complex of length exactly $d$ such that $\ell\left(H_{i}\left(C_{\bullet}\right)\right)<\infty$ for all positive $i$, then $R$ must be regular. Using Theorem 2.2, we are able to show that in the positive characteristic case, not only $k$, but also the first syzygy of $k$, cannot be a direct summand of $H_{0}\left(C_{\bullet}\right)$ for such $C_{\bullet}$ unless $R$ is regular.

Our proof of this result is quite different from that of Bridgeland and Iyengar. In particular, we avoid using the existence of big Cohen-Macaulay modules.

\section{The MAIN RESUlT}

The following fact plays a crucial role in this paper. It is contained in $[\mathrm{D}, 1.1]$. We provide a sketch of the proof here for the completeness of this paper.

Lemma 2.1. Let $(R, \mathfrak{m}, k)$ be a d-dimensional local ring of characteristic $p>0$. Let $I=(\mathbf{x})$ be an ideal generated by a system of parameters $\mathbf{x}=x_{1}, \ldots, x_{d}$. Then $\mathfrak{t}_{1}(I, R)=0$.

Proof. There is a surjection

$$
H_{1}\left(\mathbf{x}^{\left[p^{n}\right]} ; R\right) \rightarrow \operatorname{Tor}_{1}\left(R / I, f^{n} R\right)
$$

and it is well known (see $[\mathrm{R}, 7.3 .5]$ or $[\mathrm{D}, 1.1]$ ) that

$$
\lim _{n \rightarrow \infty} \ell\left(H_{i}\left(\mathbf{x}^{\left[p^{n}\right]} ; R\right)\right) / p^{n d}=0, \text { for } i>0 .
$$

The following is the main theorem of this paper.

Theorem 2.2. Let $(R, \mathfrak{m}, k)$ be a d-dimensional local ring of characteristic $p>0$. Then

(a) $e_{H K}(R)-1 \leq \mathfrak{t}_{1}(R)$;

(b) $\mathfrak{t}_{1}(R)-e_{H K}(R)+1 \leq \mathfrak{t}_{2}(R)$. 
Moreover, the following are equivalent:

(i) $R$ is regular,

(ii) $\mathfrak{t}_{1}(R)=0$,

(iii) $\mathfrak{t}_{2}(R)=0$,

(iv) $e_{H K}(R)-1=\mathfrak{t}_{1}(R)$.

Proof. We first prove (a) and (b). Let $q$ be a power of $p$. Let $I$ be an m-primary ideal generated by a system of parameters of $R$. Consider the following filtration:

$$
\begin{gathered}
0 \rightarrow Q_{1} \rightarrow R / I^{[q]} \rightarrow k \rightarrow 0, \\
0 \rightarrow Q_{2} \rightarrow Q_{1} \rightarrow k \rightarrow 0, \\
\vdots \\
0 \rightarrow k \rightarrow Q_{l} \rightarrow k \rightarrow 0 .
\end{gathered}
$$

Applying $-\otimes f^{n} R$ to the above short exact sequences, we obtain the long exact sequences

$$
\begin{aligned}
& \cdots \longrightarrow \operatorname{Tor}_{2}\left(k, f^{n} R\right) \longrightarrow \operatorname{Tor}_{1}\left(Q_{1}, f^{n} R\right) \longrightarrow \operatorname{Tor}_{1}\left(R / I^{[q]}, f^{n} R\right) \\
& \longrightarrow \operatorname{Tor}_{1}\left(k, f^{n} R\right) \longrightarrow F^{n}\left(Q_{1}\right) \longrightarrow F^{n}\left(R / I^{[q]}\right) \longrightarrow F^{n}(k) \longrightarrow 0, \\
& \cdots \longrightarrow \operatorname{Tor}_{2}\left(k, f^{n} R\right) \longrightarrow \operatorname{Tor}_{1}\left(Q_{2},{ }^{n} R\right) \longrightarrow \operatorname{Tor}_{1}\left(Q_{1}, f^{n} R\right) \\
& \longrightarrow \operatorname{Tor}_{1}\left(k, f^{n} R\right) \longrightarrow F^{n}\left(Q_{2}\right) \longrightarrow F^{n}\left(Q_{1}\right) \longrightarrow F^{n}(k) \longrightarrow 0, \\
& \cdots \longrightarrow \operatorname{Tor}_{2}\left(k, f^{n} R\right) \longrightarrow \operatorname{Tor}_{1}\left(k, f^{n} R\right) \longrightarrow \operatorname{Tor}_{1}\left(Q_{l},{ }^{n} R\right) \\
& \longrightarrow \operatorname{Tor}_{1}\left(k, f^{n} R\right) \longrightarrow F^{n}(k) \longrightarrow F^{n}\left(Q_{l}\right) \longrightarrow F^{n}(k) \longrightarrow 0 .
\end{aligned}
$$

It follows that

$$
\left(\ell\left(R / I^{[q]}\right)-1\right) \cdot \ell\left(\operatorname{Tor}_{1}\left(k, f^{n} R\right)\right)+\ell\left(F^{n}\left(R / I^{[q]}\right)\right) \geq \ell\left(R / I^{[q]}\right) \cdot \ell\left(F^{n}(k)\right)
$$

and

$$
\left(\ell\left(R / I^{[q]}\right)-1\right) \cdot \ell\left(\operatorname{Tor}_{2}\left(k, f^{n} R\right)\right)+\ell\left(\operatorname{Tor}_{1}\left(R / I^{[q]}, f^{n} R\right)\right)+\ell\left(R / I^{[q]}\right) \cdot \ell\left(F^{n}(k)\right)
$$

$$
\geq \ell\left(R / I^{[q]}\right) \cdot \ell\left(\operatorname{Tor}_{1}\left(k,{ }^{n} R\right)\right)+\ell\left(F^{n}\left(R / I^{[q]}\right)\right) .
$$

Divide both sides of (1) by $p^{n d}$ and let $n \rightarrow \infty$ to obtain

$$
\left(\ell\left(R / I^{[q]}\right)-1\right) \cdot \mathfrak{t}_{1}(R)+q^{d} \cdot e_{\mathrm{HK}}(I) \geq \ell\left(R / I^{[q]}\right) e_{\mathrm{HK}}(R) .
$$

Dividing (3) by $q^{d}$ and letting $q \rightarrow \infty$ then yields

$$
e_{\mathrm{HK}}(I) \cdot \mathfrak{t}_{1}(R)+e_{\mathrm{HK}}(I) \geq e_{\mathrm{HK}}(I) \cdot e_{\mathrm{HK}}(R) .
$$

Hence

$$
e_{\mathrm{HK}}(R) \leq 1+\mathfrak{t}_{1}(R)
$$


Similarly, from inequality (2) we can get (we need to apply Lemma 2.1 here)

$$
\left(\ell\left(R / I^{[q]}\right)-1\right) \cdot \mathfrak{t}_{2}(R)+\ell\left(R / I^{[q]}\right) e_{\mathrm{HK}}(R) \geq \ell\left(R / I^{[q]}\right) \cdot \mathfrak{t}_{1}(R)+q^{d} \cdot e_{\mathrm{HK}}(I) .
$$

Therefore

$$
\mathfrak{t}_{1}(R)-e_{\mathrm{HK}}(R)+1 \leq \mathfrak{t}_{2}(R) .
$$

For the second part of the theorem, it is clear that (i) $\Rightarrow$ (ii), (iii) and (iv) due to the exactness of Frobenius. We now prove (ii) $\Rightarrow$ (i) and (iii) $\Rightarrow$ (iv) $\Rightarrow$ (ii).

(ii) $\Rightarrow($ i). Note that inequality (3) is valid for any m-primary ideal $I$ (not just for ideals generated by a system of parameters). Since $\mathfrak{t}_{1}(R)=0$, inequality (3) becomes the equality

$$
q^{d} \cdot e_{\mathrm{HK}}(I)=\ell\left(R / I^{[q]}\right) e_{\mathrm{HK}}(R) .
$$

Taking $I=\mathfrak{m}$ immediately gives $q^{d}=\ell\left(R / \mathfrak{m}^{[q]}\right)$, which forces $R$ to be regular by Kunz's Theorem $[\mathrm{K}]$.

(iii) $\Rightarrow$ (iv). Since $\mathfrak{t}_{2}(R)=0$, inequality (4) becomes the equality

$$
\ell\left(R / I^{[q]}\right) e_{\mathrm{HK}}(R)=\ell\left(R / I^{[q]}\right) \cdot \mathfrak{t}_{1}(R)+q^{d} \cdot e_{\mathrm{HK}}(I) .
$$

We therefore obtain (iv) by dividing both sides by $q^{d}$ and taking the limits.

(iv) $\Rightarrow$ (ii). We can make a flat extension of $R$ to assume that $k$ is infinite without changing any of the relevant lengths. Let $I$ be a minimal reduction of $\mathfrak{m}$ which is generated by a system of parameters of $R$. In this case, it is well known (see, for instance, [Ma, 14.12]) that the Hilbert-Kunz multiplicity $e_{\mathrm{HK}}(I)$ coincides with the Hilbert-Samuel multiplicity $e(R)$. Taking $q=1$ in (3), we have

$$
(\ell(R / I)-1) \cdot \mathfrak{t}_{1}(R)+e_{\mathrm{HK}}(I) \geq \ell(R / I) e_{\mathrm{HK}}(R) .
$$

Replacing $e_{\mathrm{HK}}(R)$ by $1+\mathfrak{t}_{1}(R)$ in $(5)$, we get

$$
e(R) \geq \ell(R / I)+\mathfrak{t}_{1}(R) \geq \ell(R / I) .
$$

On the other hand, since $I$ is a minimal reduction of $\mathfrak{m}, e(R) \leq \ell(R / I)$. This forces all the inequalities in (6) to be equalities. Therefore $\mathfrak{t}_{1}(R)=0$.

The inequalities (a) and (b) in Theorem 2.2 are far from being the best possible bounds for the Hilbert-Kunz multiplicity. For example, the following corollary gives better bounds when $R$ is Cohen-Macaulay.

Corollary. Let $(R, \mathfrak{m})$ be a Cohen-Macaulay local ring of characteristic $p>0$ and let $e=e(R)$ be the Hilbert-Samuel multiplicity of $R$. Then

$$
e_{H K}(R)-1 \leq\left(\frac{e-1}{e}\right) \mathfrak{t}_{1}(R) .
$$

This follows easily from inequality (3) in the proof of Theorem 2.2. One can again assume the residue field of $R$ is infinite so that there is a minimal reduction $I$ of $\mathfrak{m}$ which is generated by a system of parameters. Since $R$ is Cohen-Macaulay, we have

$$
e=\ell(R / I)=e_{\mathrm{HK}}(I) .
$$

Take $q=1$ in (3) and then replace $\ell(R / I)$ and $e_{\mathrm{HK}}(I)$ in (3) by $e$. We obtain

$$
(e-1) \cdot \mathfrak{t}_{1}(R)+e \geq e \cdot e_{\mathrm{HK}}(R),
$$

which gives the desired inequality. 
Remark. When $R$ is Cohen-Macaulay, we can argue exactly the same way as in the proof of Theorem 2.2 to trivially generalize Theorem 2.2 to cases of higher Tor. Namely, we have

$$
\mathfrak{t}_{i}(R)-\mathfrak{t}_{i-1}(R)+\cdots+(-1)^{i-1} \mathfrak{t}_{1}(R)+(-1)^{i} e_{\mathrm{HK}}(R)+(-1)^{i+1} \geq 0 \text { for all } i \geq 1
$$

and $R$ being regular can be characterized by either the above inequalities taking "=" for some $i \geq 1$, or $\mathfrak{t}_{i}(R)$ being zero for some $i \geq 1$. However, the author does not know if this generality can be true without the Cohen-Macaulay assumption. The main obstruction here is, when $R$ is not Cohen-Macaulay, we no longer have the higher Tor (for $i \geq 2$ ) analog of Lemma 2.1.

\section{An IMPROVEMENT OF BRIDGELAND-IYENGAR'S RESUlT}

Recently, Bridgeland and Iyengar [B-I, 1.1] proved the following characterization for regular local rings.

Theorem 3.1 (Bridgeland-Iyengar). Let $(R, \mathfrak{m}, k)$ be a d-dimensional local ring containing a field or of dimension $\leq 3$. Assume $C_{\bullet}$ is a complex of free $R$ modules with $C_{i}=0$ for $i \notin[0, d]$, the $R$-module $H_{0}\left(C_{\bullet}\right)$ is finitely generated, and $\ell\left(H_{i}\left(C_{\bullet}\right)\right)<\infty$ for $i>0$. If $k$ is a direct summand of $H_{0}\left(C_{\bullet}\right)$, then $R$ is regular.

Their proof of Theorem 3.1 uses the existence of balanced big Cohen-Macaulay modules. Here we can apply Theorem 2.2 to give a more direct proof in the positive characteristic case that avoids using the existence of big Cohen-Macaulay modules. Moreover, our proof also yields the same conclusion if (instead of $k$ ) the first syzygy module of $k$ is a direct summand of $H_{0}\left(C_{\bullet}\right)$.

Theorem 3.2. Let $(R, \mathfrak{m}, k)$ be a d-dimensional local ring of characteristic $p>0$, $C$. a complex of free $R$-modules with $C_{i}=0$ for $i \notin[0, d]$, the $R$-module $H_{0}\left(C_{\bullet}\right)$ finitely generated, and $\ell\left(H_{i}\left(C_{\bullet}\right)\right)<\infty$ for $i>0$. If either $k$ or the first syzygy of $k$ is a direct summand of $H_{0}\left(C_{\bullet}\right)$, then $R$ is regular.

Proof. By the same argument as in [B-I, Lemma 2.2], we have a surjection

$$
H_{1}\left(F^{n}\left(C_{\bullet}\right)\right) \rightarrow \operatorname{Tor}_{1}\left(H_{0}\left(C_{\bullet}\right),{ }^{f^{n}} R\right) .
$$

It is well known that $\lim _{n \rightarrow \infty} \ell\left(H_{1}\left(F^{n}\left(C_{\bullet}\right)\right)\right) / p^{n d}=0$; see $[\mathrm{D}, 1.7]$. So we are done by Theorem 2.2 .

Remark. Theorem 3.2 is still valid for rings containing a field or of dimension $\leq 3$ (the exact same hypothesis on $R$ as in Theorem 3.1) although the proof requires the use of big Cohen-Macaulay modules. To see this, one needs to use a result of Schoutens [Sc, Proposition 2.5] to modify the original proof of Bridgeland and Iyengar (their proof of [B-I, Theorem 2.4]) slightly. We leave the details here to the readers.

It seems that the mixed characteristic case of the above result remains unknown.

\section{ACKNOWLEDGMENTS}

The author wishes to thank Graham Leuschke and Claudia Miller for introducing the paper of Bridgeland and Iyengar and for useful discussions, and to thank C.-Y. Jean Chan, who had carefully read an earlier version of this paper and suggested many improvements. 


\section{REFERENCES}

[B-E] M.Blickle and F. Enescu, On rings with small Hilbert-Kunz multiplicity, Proc. Amer. Math. Soc., 132, 2004, no. 9, 2505-2509. MR2054773 (2005b:13029)

[B-I] T. Bridgeland and S. Iyengar, A criterion for regularity of local rings, C. R. Math. Acad. Sci. Paris, 342, 2006, no. 10, 723-726. MR2227747 (2007b:13017)

[D] S. P. Dutta, Ext and Frobenius, J. Algebra, 127, 1989, 163-177. MR1029410 (91g:13018)

[H-Y] C. Huneke and Y. Yao, Unmixed local rings with minimal Hilbert-Kunz multiplicity are regular, Proc. Amer. Math. Soc., 130, 2002, no. 3, 661-665. MR1866016 (2002h:13026)

[K] E. Kunz, Characterization of regular local rings for characteristic p, Amer. J. Math., 91 1969, 772-784. MR0252389 (40:5609)

[Ma] H. Matsumura, Commutative Ring Theory, Cambridge Stud. Adv. Math., 8, Cambridge Univ. Press, Cambridge, 1986. MR879273 (88h:13001)

[Mo] P. Monsky, The Hilbert-Kunz function, Math. Ann., 263, 1983, 43-49. MR697329 (84k:13012)

[R] P. Roberts, Multiplicities and Chern Classes in Local Algebra, Cambridge University Press, 1998. MR1686450 (2001a:13029)

[Sc] H. Schoutens, On the vanishing of Tor of the absolute integral closure, J. Algebra, 275, 2004, 567-574. MR2052626 (2005g:13010)

[Se] G. Seibert, Complexes with homology of finite length and Frobenius functors, J. Algebra, 125, 1989, 278-287. MR1018945 (90j:13012)

[W-Y] K.-I. Watanabe and K. Yoshida, Hilbert-Kunz multiplicity and an inequality between multiplicity and colength, J. Algebra, 230, 2000, 295-317. MR1774769 (2001h:13032)

Department of Mathematics, Syracuse University, 215 Carnegie, Syracuse, New York 13244

E-mail address: jli32@syr.edu 